\title{
Radio Frequency Power Sensor Based on MEMS Technology with Ultra LOW LOSSES
}

\author{
Luis Fernández, Javier Sesé, Remco Wiegerink, Jaap Flokstra, Henri Jansen and Miko Elwenspoek \\ MESA+ Institute, University of Twente, P.O. Box 217, 7500 AE Enschede, The Netherlands
}

\begin{abstract}
A wideband $100 \mathrm{kHz}-4 \mathrm{GHz}$ power sensor, of which the basic design was presented recently [1], was successfully realized. The sensor is based on sensing the electrical force between the RF signal line and a suspended membrane. Optimization of the design with SONNET has resulted in measured reflection losses (S11) less than $-30 \mathrm{~dB}$, transmission losses ( $\mathrm{S} 12)$ better than $-0.2 \mathrm{~dB}$, and a sensitivity of $80 \mathrm{aF} / \mathrm{mW}$.
\end{abstract}

\section{INTRODUCTION}

Recently a new sensing principle was proposed for measuring RF signal power [2-5]. This principle is based on sensing the attractive electrical force between the signal line and a grounded electrode that is suspended at a small distance from the signal line. A high frequency power sensor based on this principle has very interesting properties. Most important is the possibility to operate the same sensor from low frequency $\mathrm{AC}$ through to microwaves as a true wideband device extending the frequency range of available instrumentation (voltmeters, power meters, signal sources). Furthermore, the sensor does not absorb the signal. Instead, a "through" sensor is realized, with almost no loss of signal. Most existing technology for measuring the power of RF signals is based on terminating devices, i.e. the power is lost after the measurement. Examples are microcalorimeters, bolometers, and thermistor mounts. These devices suffer from sensitivity to ambient temperature changes, limited dynamic range and long settling times. Diode sensors provide the ability to measure the envelope and modulation and have the advantage of greater dynamic range, but they suffer from temperature sensitivities and problems with impedance matching. These sensors cannot easily be integrated with signal conditioning or measurement electronics.

\section{PRINCIPLe of OPERATION}

The operation of the sensor is based on the attractive force between the two plates of a charged parallel plate capacitor. An ac voltage between the plates results in a timedependent, but always attractive, electrical force. For frequencies well above the mechanical resonance frequency, only the average force is important, which can be expressed as:

$$
F_{e l}=\frac{1}{2} \frac{C}{x} u_{R M S}^{2}
$$

with $C$ the capacitance, $x$ the distance between the plates, and $u_{R M S}$ the RMS voltage amplitude of the signal. Thus, if one of the capacitor plates is moveable, the (square of the) RMS voltage amplitude can be obtained by measuring the displacement of the moveable capacitor plate or by measuring the force needed to keep the plate in its initial position. This effect is well known and has been exploited to realize micro-mechanical RMS-to-DC converters [6-8].

The idea to exploit this principle at very high frequencies, i.e. in the GHz range, was presented by Seppä et al. [2]. There are several reasons why such a sensor has not been realized before. Of course, the sensor design becomes much more complicated, because one of the capacitor electrodes has to be replaced by a high-frequency transmission line. Furthermore, due to the currents in such a transmission line there will also be a magnetic force, which opposes the electrical force. In fact, in an ideal, lossless transmission line the magnetic force exactly cancels the electric force. Finally, such high frequencies require the use of materials with low dielectric losses. For example, the device presented in this paper was realized by aluminum surface micromachining on a substrate of AF45 glass.

Fig. 1 shows a schematic drawing of the basic sensor structure. Two sensing electrodes are integrated between the signal line and ground lines of a coplanar waveguide (CPW). A membrane is suspended above the electrodes and fixed to the ground lines. An RF signal on the centerline causes a downward force on the (grounded) membrane. The resulting deflection of the membrane is measured by measuring the capacitance between the membrane and the sense electrodes.
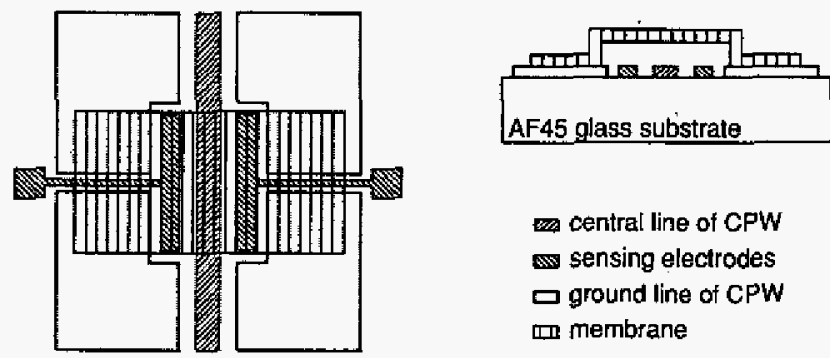

central line of CPW sensing electrodes $\square$ ground line of CPW In membrane

Fig. 1: Schematic drawing of the sensor structure, left: top view, right: cross sectional view.

\section{DESIGN}

The first prototype sensors [4] had rather poor RF properties and low sensitivity. Therefore, some improvements were proposed in [1]. Here we will focus on 
three aspects: the RF properties of the sensor, the mechanical properties of the moveable plate suspension, and the capacitive read-out mechanism that was chosen for detecting the displacement of the moveable plate.

\section{RF design}

As mentioned above, the sensor basically consists of a coplanat waveguide (CPW) with a moveable plate suspended above the signal line. The width of the signal line is $100 \mu \mathrm{m}$ and the distance between the signal line and ground lines is $25 \mu \mathrm{m}$, in order to obtain a characteristic impedance of $50 \Omega$. The insertion of a capacitor in the transmission line will inevitably lead to reflections and transmission losses. As long as the dimensions of the added capacitance are small compared to the signal wavelength, it can be considered as a lumped element. In that case, at the position of the capacitor we have an impedance mismatch, and the reflected power can be expressed as:

$$
P_{R}=\left|\frac{Z_{L}-Z_{0}}{Z_{L}+Z_{0}}\right|^{2} P_{T}
$$

where $P_{T}$ is the total signal power, $Z_{0}$ is the characteristic impedance of the transmission line before the discontinuity and $Z_{L}$ is the impedance due to the presence of the extra capacitance.

For a large bandwidth the capacitance should be as small as possible. On the other hand, for maximum sensitivity a large capacitance is needed. Adjusting the CPW impedance directly before and after the sensor capacitance can relieve this trade-off. Two techniques were considered: a) changing the width of the signal line (Fig. $2(a)$ ), and b) increasing the gap size of the CPW (Fig. 2(b)). In both cases the area between the signal line and the ground is increased resulting in a higher inductance, which counter balances the extra capacitance of the sensor structure. In this way, a better impedance match is expected. Optimal dimensions were found using SONNET simulations. Devices with various dimensions for the length (d) and width (g) of the gaps were realized for comparison with the simulation results,

\section{Moveable capacitor plate}

The moveable capacitor plate is realized by aluminum surface micromachining using photoresist as sacrificial layer. A clamped-clamped beam or bridge configuration was chosen in order to minimize the deformation of the bridge due to stress gradients in the aluminum layer. Furthermore, the electrodes under the bridge are buried in the substrate in order to obtain a very flat bridge structure. In this way, the initial gap of the capacitor is very well predicted. In order to be sensitive to low RF power ( $m$ Watt range) a low spring constant is needed. Therefore, the bridge was dimensioned $900 \mu \mathrm{m}$ long, $100 \mu \mathrm{m}$ wide and 1 $\mu \mathrm{m}$ thick, getting close to the limits of our fabrication process.

\section{Power read-out design}

A capacitive readout was chosen to measure the displacement of the bridge because of its straightforward implementation and high sensitivity. Capacitance changes in the order of $0.1 \mathrm{fF}$ are easily measured by commercial systems (HP 4192). With an initial gap distance of $1 \mu \mathrm{m}$ and $0.1 \mathrm{pF}$ capacitance displacements in the order of $1 \mathrm{~nm}$ can be detected. Furthermore, changes in the order of $1 \mathrm{aF}$ can be detected by dedicated electronics placed close to the sensor component.

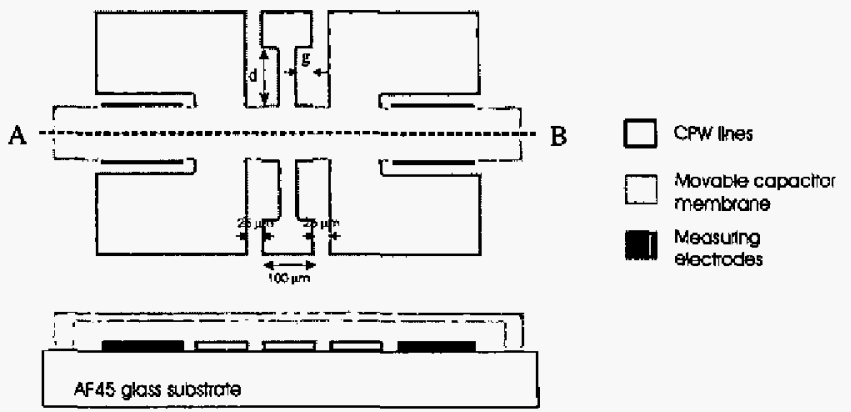

(a)
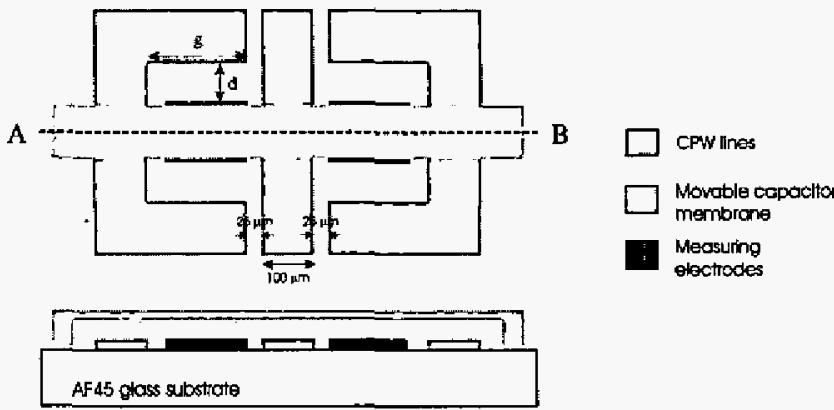

(b)

Fig. 2: Schematic drawings for two different techniques to improve the RF properties of the sensor: a) changing the width of the signal line, and b) increasing the gap size of the CPW.

\section{Fabrication}

Since the capacitance added to the CPW needs to be very well defined, special care has to be taken in the fabrication of the moveable capacitor. One of the most important problems is to obtain a flat membrane over the CPW. The thickness of the CPW lines is similar to the thickness of the sacrificial layer used for the gap definition and, therefore, no good planarization of the sacrificial layer can be achieved. As a result, the shape of the CPW electrodes is reflected in the membrane, which in combination with some residual stress will result in deformation of the membrane. In order to avoid this problem, a fabrication process was used where CPW and sense electrodes are embedded in the substrate,

Fig. 3 shows a summary of the fabrication scheme. First, a $1 \mu \mathrm{m}$ deep recess is etched using a photoresist mask. Then, $900 \mathrm{~nm}$ of aluminum is deposited by sputtering. Removing the photoresist results in lift-off of the aluminum layer, giving an almost flat substrate with the coplanar waveguide and sense electrodes embedded in the surface. Finally, the moveable plate is realized by aluminum surface micromachining using photoresist as sacrificial layer. Fig. 4 
and Fig. 5 show SEM photographs of realized devices. The close-up in Fig. 4 shows that the embedding of the lower electrodes in the substrate indeed results in a very flat moveable plate. Fig. 5 shows SEM pictures of the two different designs of RF power sensors.

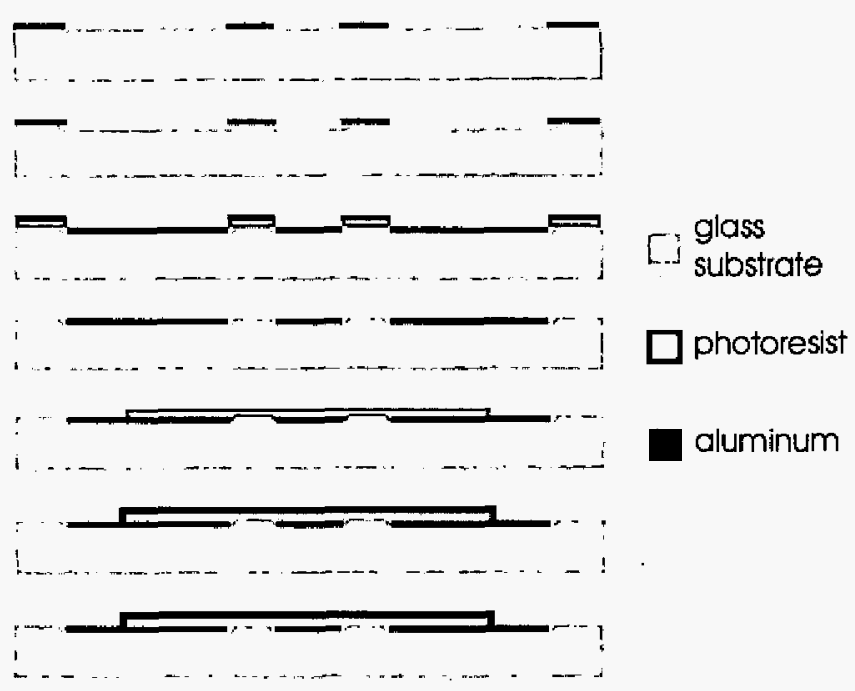

Fig. 3: Fabrication scheme of 'the $r f$ power sensor for realization of flat moveable capacitor

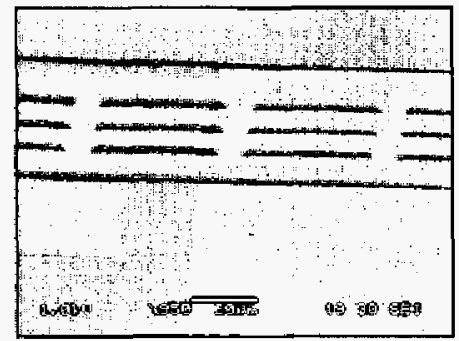

Fig. 4: Close up of the moveable plate above the lower electrodes embedded in the substrates.

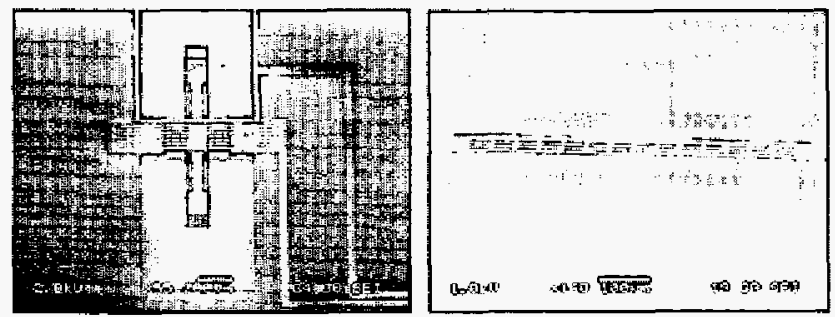

Fig. 5: SEM pictures of the two different designs of RF power sensors as depicted in Fig. 2.

\section{RESULTS}

The RF performance of the power sensor was characterized by measuring the reflection losses $S 11$ and transmission losses $\mathrm{S} 12$ for frequencies up to $4 \mathrm{GHz}$, and the sensitivity was measured by detecting the capacitance change as a function of the applied RF power at various frequencies.

\section{S-parameters measurement}

An HP85 10C Vector Network Analyzer was used for the Sparameters measurements of the two different designs with different dimensions. Measurements were performed up to $4 \mathrm{GHz}$. Fig. 6 shows the results for the design in which the gap between the central line and the ground planes $(\mathrm{g})$ is modified by reducing the width of the signal line along a certain distance (d) (Fig. 2(a)). Lower reflection losses are obtained when $\mathrm{g}$ and $\mathrm{d}$ are increased, but also the transmission losses increase. The latter is due to the higher resistance that results from the reduced width of the signal line. We can conclude that optimization of the reflection losses can be obtained by this design, but no optimization of transmission losses is possible.

Fig. 7 shows the measurement results of the sensor design where the gap distance $(\mathrm{g})$ is increased by placing the ground lines further away along a certain distance (d) (Fig. 2(b)). Again the reflection losses improve by almost $10 \mathrm{~dB}$ at $4 \mathrm{GHz}$., but now also the transmission losses improve. Only for the extreme situation with $\mathrm{d}=250 \mu \mathrm{m}$ and $\mathrm{g}=175 \mu \mathrm{m}$ and we see that the transmission losses get larger again. This is due to increased resistive losses in the ground lines (with a higher value of $g$ the width of the ground lines decreases). We can conclude that both reflection and transmission losses can be optimized by adjusting the dimensions $\mathrm{g}$ and $\mathrm{d}$.

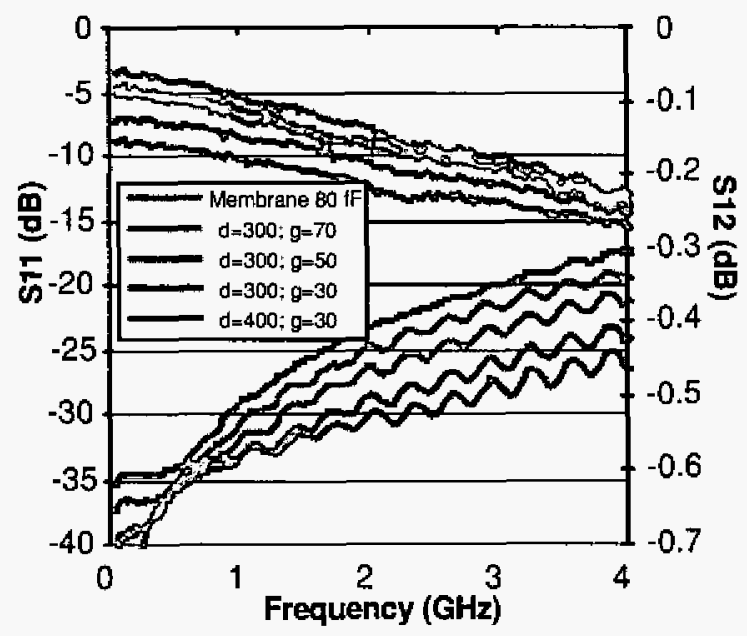

Fig. 6: S-parameter measurements for the sensor design where the width of the signal line is reduced (Fig. 2(a)).

\section{Power measurements}

Power measurements were done by measuring the capacitance between one of the sense electrodes and the moveable membrane as a function of the power of an RF signal on the signal line. For the capacitance measurement, an HP 4195 Impedance Analyzer was used. The capacitance added to the line by the moveable capacitor was measured to be $0.1 \mathrm{pF}$, while the total capacitance measured on the sensing capacitor was $0.4 \mathrm{pF}$, both as expected from geometrical considerations: Capacitance as a function of power level of the RF signal at different 
frequencies was measured. The results are presented in Fig. 8.

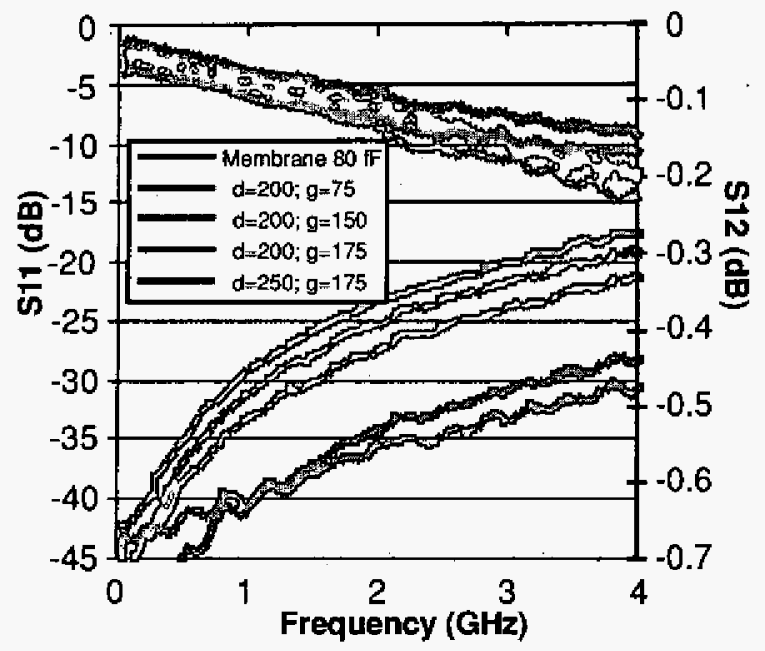

Fig. 7: S-parameter measurements for the sensor design where the distance between ground lines and signal line is increased (Fig. 2(b)).

As expected, a linear relation between measured capacitance and power level of the RF signal was detected. The small difference found in the response of the sensor for different frequencies of the RF signal is mainly due to the reflection dependency of the cables due to their length of around $30 \mathrm{~cm}$ and their own frequency dependent transmission losses. From Fig. 8 we can conclude that a resolution in the order of $\mathrm{mW}$ is obtained. Using dedicated electronics for the capacitance measurement, a resolution down to microwatts should be feasible.

\section{CONCLUSIONS}

A wideband $100 \mathrm{kHz}-4 \mathrm{GHz}$ power sensor was successfully realized. The sensor is based on sensing the electric force between the RF signal line and a suspended membrane. It was shown that a slight adjustment of the waveguide just before and after the sensor capacitance reduces the reflection losses by $10 \mathrm{~dB}$ to $-30 \mathrm{~dB}$ at $4 \mathrm{GHz}$. The transmission losses are dominated by resistive losses in the coplanar waveguide. The capacitance change as a function of applied RF power was measured at several frequencies. The sensitivity was in the order of $80 \mathrm{aF} / \mathrm{mW}$. Some frequency dependence was observed, but this is expected to be due to the measurement setup.

\section{ACKNOWLEDGEMENTS}

This work is part of the IST-2000-28261 EMMA program of the European Commission. We wish to thank Tauno Vähä-Heikkilä, Jukka Kyynäräinen, Aarne Oja, Jussi Varis, Heikki Seppä and Anu Kärkkäinen from VTT for useful discussions. Furthermore, we wish to thank Paul Roberts and Simon Hollingworth from Fluke for their help with the packaging study of the sensor.

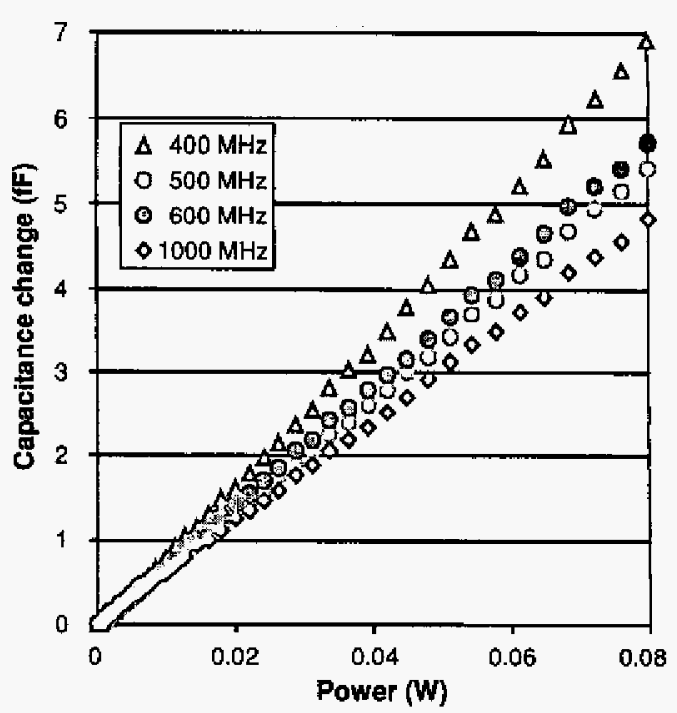

Fig. 8: Capacitance change measured as a function of the power level of the of signal traveling through the sensor at 4 different frequencies $(400,500,600$ and $1000 \mathrm{MHz})$.

\section{REFERENCES}

[1] L.J. Fernandez, E. Visser, J, Sesé, R.J. Wiegerink, H.V. Jansen, J. Flokstra, and M. Elwenspoek, "Development of a capacitive MEMS RF power sensor without dissipative losses: towards a new philosophy of RF power sensing," CPEM 2004, London, UK, June 27 - July 2, 2004.

[2] H. Seppä, J. Kyynäräinen, A. Oja, "Microelectromechanical systems in electrical metrology", IEEE Trans. Instrum. and Meas., 50, pp. 440-444, 2000.

[3] T. Vähä-Heikkilä, J. Kyynäräinen, A. Oja, J. Varis, and H. Seppä, "Capacitive MEMS power sensor", MEMSWAVE, Crete, 2002.

[4] L.J. Fernandez, E. Visser, J. Sesé, R.J. Wiegerink, H.V. Jansen, J. Flokstra, and M. Elwenspoek, "Capacitive if power sensor based on MEMS technology," IEEE Sensors Conference, Toronto, October 22-24, 2003.

[5] Ari Alastalo, Jukka Kyynäräinen, Heikki Seppä, Anu Kärkkäinen, Nadine Pesonen ,Manu Lahdes, Tauno Văhä-Heikkilä , Panu Pekko and James Dekker, "Wideband Microwave Power Sensor Based on MEMS Technology", CPEM 2004, London, UK, June 27 - July 2, 2004.

[6] B. P. Drieënhuizen and R. F. Woffenbuttel, "Integrated electrostatic true RMS-to-DC converter", IEEE Transactions on Instrumentation and Measurement, 44, 1995, pp. 370-373.

[7] M. Suhonen, H. Seppä, A.S. Oja, M. Heinilä and I. Näkki, "AC and DC Voltage Standards Based on Silicon Micromechanics", Proceedings CPEM, Washington DC, 1998, pp. 23-24.

[8] M. Bartek, Z. Xiao, C. van Mullem and R.F. Wolffenbuttel, Bulk-micromachined electrostatic RMS-to-DC converter: Design and fabrication, Proceedings MME, Uppsala, Sweden, October 1-3, 2000. 